\section{Colitis de Crohn: resultados del tratamiento quirúrgico y evolución alejada}

\author{
FELIPE IMIGO G. ${ }^{1}$, MARÍA-ELENA MOLINA P. ${ }^{1}$, \\ MANUEL ÁLVAREZ-LOBOS ${ }^{2}$, CARLOS QUINTANA V. ${ }^{2}$, \\ JULIETA KLAASSEN L. ${ }^{1}$, JAVIERA TORRES M. ${ }^{3}$, IGNACIO DUARTE G. ${ }^{3}$, \\ FELIPE BELLOLIO R. ${ }^{1}$, ÁLVARO ZÚÑIGA D. ${ }^{1}$
}

\section{Outcome of surgery for Crohn's colitis. Review of 28 cases}

Background: Exclusive involvement of the colon or rectum in Crohn's disease, called Crohn's colitis, (CC) occurs in about 25\% of these patients. Aim: To analyze early surgical results and long-term outcomes of patients undergoing surgery for CC. Material and Methods: Review of a prospective database, identifying patients with Crohn's disease operated between 2003 and 2015 and excluding those with ileocecal disease. We analyzed demographic data, pre and postoperative pharmacological treatment, operations, morbidity and the need for a second bowel resection at follow-up. Results: We reviewed data from 28 patients aged 17 to 72 years (15 men). Twenty-seven (96.4\%) had previous pharmacological treatment, 11 received monoclonal antibodies. The most common indications for surgical treatment were failure of medical treatment in 15 cases, acute severe colitis in 12 and anemia/malnutrition in eight. Total colectomy was performed in 17 (61\%) patients, proctocolectomy in $8(29 \%)$ and segmental colectomies in 3 (11\%). Sixteen (57\%) were operated laparoscopically. Major postoperative complications were observed in 5 (18\%). Four needed a reintervention. There was no operative mortality. During a 55 months median follow-up of 27 patients, seven (26\%) required a second bowel resection, one of them for recurrence. Nineteen (70\%) patients had an ostomy, which was permanent in 11. Fifteen patients are without medical treatment. Conclusions: Most of the reviewed patients required total colectomy for the control of the disease with a low surgical morbidity. Two-thirds required an ileostomy, which became permanent in half of them.

(Rev Med Chile 2018; 146: 183-189)

Key words: Colitis; Colorectal Surgery; Crohn Disease.

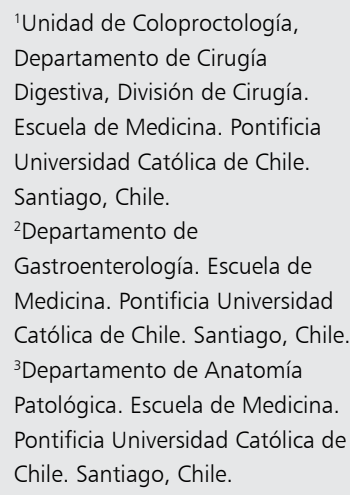

Financiamiento: Financiamiento propio por los autores sin fuente externa.

Recibido el 6 de junio de 2017, aceptado el 6 de febrero de 2018.

Correspondencia a:

Dr. Felipe Bellolio Roth Diagonal Paraguay 362 Edificio Académico,

Pontificia Universidad Católica de Chile. $4^{\circ}$ piso.

Departamento de Cirugía Digestiva. Santiago, Chile. Teléfono: 223543870 / 223543462

fbelloli@med.puc.cl
E 1 carácter crónico y panintestinal de la enfermedad de Crohn (EC) determina que los pacientes sufran con frecuencia recurrencia de la enfermedad a pesar del éxito inicial del tratamiento médico. En la EC, la localización ileocecal es la forma más frecuente de compromiso intestinal (35-40\%). Varios estudios comunican cifras de recurrencia variables entre 30 y $50 \%$ para los pacientes que han sido sometidos a una resección intestinal, en seguimientos de 10 a 15 años, incluyendo criterios diagnósticos clínicos, endoscópicos y radiológicos ${ }^{1-7}$. El compromiso exclusivo de colon o recto, colitis de Crohn (CC), se observa en alrededor de $25 \%$ de los pacientes y las cifras de recurrencia varían entre 9 y $40 \%$ luego del tratamiento quirúrgico resectivo dependiendo, en parte, del tipo de resección practicada ${ }^{1,8,9}$. Cuando la enfermedad está confinada al colon, recto o 
ambos, el desarrollo de crisis de colitis, sangrado, compromiso del estado nutricional, intolerancia al tratamiento médico, fístulas, sepsis perianal, estenosis o compromiso de la continencia son condiciones que separadamente o en conjunto constituyen indicaciones quirúrgicas ${ }^{10,11}$.

La colectomía total, la colectomía segmentaria y la proctocolectomía son las alternativas de tratamiento quirúrgico que hay que ponderar detenidamente ante la presencia simultánea de compromiso perineal en forma de fisuras, fístulas, abscesos o incontinencia. Existen pocas comunicaciones que se refieren al tratamiento quirúrgico y sus resultados en pacientes con CC sin compromiso ileocecal ${ }^{12-16}$.

El objetivo de este estudio es comunicar los resultados quirúrgicos a corto y largo plazo en una serie de pacientes con CC.

\section{Material y Métodos}

Se realizó un estudio de cohorte no concurrente, identificando en la base de datos prospectiva de enfermedades inflamatorias intestinales de los Departamentos de Cirugía Digestiva y Gastroenterología de la Escuela de Medicina de la Pontificia Universidad Católica de Chile a todos los pacientes operados con el diagnóstico preoperatorio de CC entre los años 2003 y 2015. Se excluyeron aquellos pacientes con un diagnóstico preoperatorio de colitis ulcerosa y que en su seguimiento evolucionaron como una EC y los pacientes con EC de la región ileocecal ${ }^{17}$.

Se planteó como resultado primario la morbimortalidad quirúrgica y como resultado secundario la necesidad de una segunda cirugía sobre el intestino en el período de seguimiento postoperatorio.

El manejo pre, intra y postoperatorio estuvo a cargo de un equipo multidisciplinario, con participación de gastroenterólogos, nutriólogos, radiólogos, enfermeras y coloproctólogos.

El diagnóstico estuvo basado en la combinación de las características clínicas, hallazgos endoscópicos y de imágenes, y que fue confirmado por el estudio macroscópico e histopatológico de la pieza operatoria.

Se consignaron las variables demográficas, extensión de la enfermedad, clasificación de Montreal para enfermedad de Crohn, tratamien- to farmacológico pre y postoperatorio, morbimortalidad quirúrgica según la clasificación de Clavien-Dindo y necesidad de una segunda intervención sobre el intestino en el seguimiento a largo plazo ${ }^{18,19}$. La concurrencia de una o más de las siguientes condiciones clínicas constituyeron la indicación operatoria: Fracaso de tratamiento médico, colitis en el contexto de una crisis grave, anemia/desnutrición, estenosis, presencia de fístulas, perforación de colon y hemorragia digestiva baja.

Se consideró fracaso del tratamiento médico la concurrencia de la falta de respuesta a la terapia con 5-ASA, esteroides, inmunomoduladores o biológicos, la persistencia de anemia (hematocrito $<30 \%$ ) y desnutrición (albuminemia $<3 \mathrm{~g} / \mathrm{dl}$ ).

La técnica quirúrgica empleada, laparoscópica o cirugía clásica, colectomía parcial, total o proctocolectomía fue seleccionada por el cirujano de acuerdo a las condiciones clínicas y hallazgos en la exploración abdominal.

Se definió como intervención quirúrgica de urgencia aquella operación practicada dentro de las $72 \mathrm{~h}$ después del ingreso, período empleado en corregir graves alteraciones de los parámetros fisiológicos (hipokalemia, anemia, insuficiencia renal prerrenal, etc.) en el contexto de una colitis grave. Se consideró morbilidad mayor aquellas complicaciones que requirieron un manejo activo mediante radiología intervencional o reoperación (clasificación Clavien-Dindo III o superior).

El seguimiento de los pacientes estuvo a cargo de los médicos tratantes, considerando como seguimiento alejado aquel mayor a 6 meses. El diagnóstico de recurrencia se hizo en base a parámetros clínicos, que por limitaciones económico-geográficas se confirmó endoscópicamente solo en algunos pacientes.

Los datos fueron tabulados en el programa SPSS versión 22.0 realizando estadística descriptiva de estos.

El estudio fue aprobado por el Comité de Ética de la Facultad de Medicina de la Pontificia Universidad Católica de Chile (Proyecto No 170109008).

\section{Resultados}

En el período estudiado, 28 pacientes cumplieron los criterios de inclusión con compromiso exclusivo de colon, según la clasificación de Mon- 
treal. Quince $(53,6 \%)$ fueron de sexo masculino. La mediana de edad fue de 37,5 años. Diecisiete $(60,7 \%)$ pacientes presentaban colitis extensa y 11 $(39,3 \%)$ compromiso segmentario. El recto estaba comprometido en 9 pacientes del total $(32,1 \%)$ de la serie.

La enfermedad perineal estuvo presente en $15(53,6 \%)$ pacientes, de los cuales 6 presentaban compromiso concomitante del recto. Trece $(46,4 \%)$ pacientes tenían manifestaciones extraintestinales (artralgias en 6, aftas bucales en 4, eritema nodoso en 2 y sacroileítis en 1). El tiempo de evolución de la enfermedad antes del tratamiento quirúrgico tuvo una mediana de 6 años. Las características demográficas y la clasificación de Montreal se detallan en la Tabla 1.

Veintisiete pacientes $(96,3 \%$ habían recibido tratamiento farmacológico en los 3 meses previos al tratamiento quirúrgico, dentro de ellos, los corticoides fueron los fármacos más utilizados, 64,3\% (Tabla 2).

El tratamiento quirúrgico se practicó en forma electiva en 22 pacientes $(78,6 \%)$ y de urgencia en $6(21,4 \%)$. Una o más condiciones clínicas determinaron la necesidad de tratamiento quirúrgico. Las condiciones más frecuentes fueron el fracaso del tratamiento médico, en 15 casos $(53,5 \%)$ y la colitis en el contexto de una crisis grave, en 12 $(42,8 \%)$ (Tabla 3$)$.

Las operaciones practicadas en la primera intervención quirúrgica fueron: la colectomía total con ileostomía terminal y preservación del recto en 13 pacientes $(46,4 \%)$, la proctocolectomía con ileostomía terminal definitiva en $8(28,6 \%)$, la colectomía total con ileorectoanastomosis (IRA) en $4(14,3 \%)$ y colectomía segmentaria en $3(10,7 \%)$. Uno de estos últimos pacientes presentó como hallazgo incidental en la pieza operatoria un cáncer de recto superior, por lo tanto, se completó su tratamiento con una proctocolectomía.

El abordaje laparoscópico se realizó en 16 casos $(57,1 \%)$, con necesidad de una conversión.

Complicaciones postoperatorias se registraron en 9 pacientes $(32,1 \%)$ (Tabla 4). Cinco de estas $(17,5 \%)$, Clavien-Dindo $\geq$ III, necesitaron tratamiento quirúrgico/percutáneo. En 2, con íleo mecánico, se practicó adherenciolisis y en las otras 2, con una colección intraabdominal, se realizó drenaje por punción percutánea en uno y drenaje abierto en el otro. En este último paciente, simultáneamente se practicó una proctectomía
Tabla 1. Características demográficas y clínicas de la serie según Clasificación de Montreal

\begin{tabular}{|c|c|}
\hline Variable & (\%) \\
\hline Edad (mediana/rango) & $37,5(17-72)$ \\
\hline \multicolumn{2}{|l|}{ Sexo } \\
\hline Masculino & $15(53,6)$ \\
\hline Femenino & $13(46,4)$ \\
\hline \multicolumn{2}{|l|}{$\begin{array}{l}\text { Tiempo enfermedad en años } \\
\text { (mediana/rango) }\end{array}$} \\
\hline \multirow{2}{*}{\multicolumn{2}{|c|}{$\begin{array}{l}\text { Clasificación de Montreal } \\
\text { Edad de diagnóstico }\end{array}$}} \\
\hline & \\
\hline A1 & $4(14,3)$ \\
\hline A2 & $17(60,7)$ \\
\hline A3 & $7 \quad(25)$ \\
\hline \multicolumn{2}{|l|}{ Localización enfermedad } \\
\hline L2 & $28(100)$ \\
\hline \multicolumn{2}{|l|}{ Patrón evolutivo } \\
\hline B1 & $20 \quad(71,4)$ \\
\hline B2 & $5 \quad(17,9)$ \\
\hline B3 & $3(10,7)$ \\
\hline Compromiso perineal & $15(53,6)$ \\
\hline Compromiso extraintestinal & $13(46,4)$ \\
\hline \multicolumn{2}{|l|}{ Extensión afectación en colon-recto } \\
\hline Colitis extensa & $17(60,7)$ \\
\hline Segmentario & $11(39,3)$ \\
\hline Albuminemia $<3 \mathrm{~g} / \mathrm{dl}$ & $16(57,1)$ \\
\hline Hematocrito $<30 \%$ & 7 (25) \\
\hline $\begin{array}{l}\text { Estadía preoperatoria } \\
\text { (mediana/rango) }\end{array}$ & $3,5(0-44)$ \\
\hline
\end{tabular}

Clasificación de Montreal: Edad de diagnóstico: A1 $\leq 16$ años; A2: 17-40 años; $A 3>40$ años. Localización enfermedad: L1 ileal; L2 colónica; L3 ileocólica; L4 gastrointestinal alta. Patrón evolutivo: B1 no-obstructivo no-fistulizante (inflamatorio); B2 obstructivo (fibroestenosante); B3 fistulizante. Compromiso perianal se agrega la letra $p$.

Tabla 2. Tratamiento farmacológico en los 3 meses previos al tratamiento quirúrgico

\begin{tabular}{|lc|}
\hline Fármacos & $\begin{array}{c}\text { Pacientes } \\
\text { n (\%) }\end{array}$ \\
\hline Corticoides & $18(64,3)$ \\
\hline Aminosalicilatos & $15(53,6)$ \\
\hline Inmunomoduladores* & $13(46,4)$ \\
\hline Biológicos** & $11(39,3)$ \\
\hline Antibióticos & $10(35,7)$ \\
\hline
\end{tabular}

*Azatioprina, metotrexato, 6-mercaptopurina. **9 infliximab, 2 adalimumab. 
Tabla 3. Indicaciones quirúrgicas en 28 pacientes con colitis de Crohn

\begin{tabular}{|lcc|}
\hline $\begin{array}{l}\text { Indicación } \\
\text { (1 o más por paciente) }\end{array}$ & $\begin{array}{c}\text { n (del total } \\
\text { de pacientes) }\end{array}$ & \% \\
Fracaso tratamiento médico & 15 & $(53,5)$ \\
Colitis (crisis grave) & 12 & $(42,8)$ \\
Desnutrición/anemia & 8 & $(28,5)$ \\
$\begin{array}{l}\text { Estenosis de colon } \\
\text { sigmoides/anorrectal }\end{array}$ & 5 & $(17,7)$ \\
Fístulas & 3 & $(10,7)$ \\
Perforación de colon & 2 & $(7,1)$ \\
Hemorragia digestiva baja masiva & 1 & $(3,5)$ \\
\hline
\end{tabular}

\section{Tabla 4. Complicaciones postoperatorias en 28 pacientes con colitis de Crohn sometidos a tratamiento quirúrgico}

\begin{tabular}{|lcc|}
\hline Complicación & $\begin{array}{c}\mathbf{n} \\
\text { pacientes }\end{array}$ & \% \\
\hline Infección de herida operatoria & 2 & 7 \\
\hline Colección intraabdominal & 2 & 7 \\
\hline Íleo mecánico & 2 & 7 \\
\hline íleo adinámico & 1 & 3,5 \\
\hline Hemorragia digestiva alta & 1 & 3,5 \\
\hline Perforación inadvertida íleon distal & 1 & 3,5 \\
\hline
\end{tabular}

Tabla 5. Operaciones practicadas en la primera y segunda intervención quirúrgica en 28 pacientes con colitis de Crohn

\begin{tabular}{|c|c|c|c|}
\hline \multicolumn{4}{|c|}{ Intervenciones quirúrgicas } \\
\hline $\begin{array}{l}1^{\circ} \text { operación } \\
n=28\end{array}$ & n (\%) & $\begin{array}{l}2^{\circ} \text { operación } \\
n=7\end{array}$ & $\mathbf{n}$ \\
\hline $\begin{array}{l}\text { Colectomía total + ileostomía terminal + } \\
\text { preservación rectal }\end{array}$ & $13(46,4)$ & $\begin{array}{l}\text { Íleo-recto anastomosis } \\
\text { Proctectomía }\end{array}$ & $\begin{array}{l}3 \\
2\end{array}$ \\
\hline Proctocolectomía + ileostomía definitiva & $8(28,5)$ & Resección íleon terminal por recurrencia & 1 \\
\hline Colectomía total + íleo-recto anastomosis & $4(14,2)$ & $\longrightarrow$ & 0 \\
\hline Colectomía segmentaria & $3(10,7)$ & Proctocolectomía & 1 \\
\hline
\end{tabular}

por el hallazgo incidental de un cáncer de recto superior en el estudio histopatológico de la pieza operatoria. En el paciente restante, con una perforación inadvertida del íleon terminal, se practicó una resección segmentaria.

La mediana de estadía hospitalaria fue de 18 días (rango de 5 a 78 días), destacando una estadía preoperatoria de 3,5 (rango de 0 a 44 días) y una estadía postoperatoria de 13,5 (rango de 4 a 63 días).

En el subgrupo de 9 pacientes con estadía prolongada (preoperatoria mayor a 4 días y postoperatoria mayor a 14), 8 presentaban hipoalbuminemia y habían recibido esteroides previos a la hospitalización. Cinco tenían un hematocrito $<30 \%$. En estos pacientes, la operación practicada con mayor frecuencia fue la colectomía total con preservación del recto e ileostomía terminal, en 5 casos.
No hubo mortalidad operatoria en la serie.

El seguimiento fue completo en 27 de los 28 pacientes (96,4\%), con una mediana de 55 meses (rango 2 a 160 meses). En la evolución alejada, 7 pacientes $(25 \%)$ requirieron una segunda operación sobre el intestino. En 3 de ellos para practicar una IRA, en 3 una proctectomía y una resección segmentaria de íleon terminal por recurrencia en un paciente, previamente sometido por una proctocolectomía. Todas ellas, excepto una, después de 6 meses luego de la primera operación (rango de 1-76 meses) (Tabla 5).

Al término del período de seguimiento, 11 $(40,7 \%)$ pacientes tenían una ileostomía definitiva y $7(25,9 \%)$ una IRA. Ocho $(29,6 \%)$ tenían una ileostomía transitoria, de los cuales 4 presentan enfermedad rectoperineal activa, 2 habían rechazado la reconstitución del tránsito y 2 pacientes esperaban una IRA. 
En relación al tratamiento farmacológico, este disminuyó, siendo necesario en 12 de los 27 pacientes iniciales $(44,4 \%)$, sin existir una diferencia significativa entre estos grupos $\left(\chi^{2}, p=0,44\right)$.

Un paciente falleció alejadamente por evolución de su enfermedad neoplásica (cáncer de recto).

\section{Discusión}

En el análisis de los resultados de esta serie es satisfactorio comprobar que el tratamiento quirúrgico de la CC en nuestra institución es factible llevarlo a cabo con resultados de morbilidad similares a las de otras series, entre 23 y $26 \%{ }^{3,8}$.

Los resultados favorables observados en esta serie pueden explicarse como efecto del trabajo de un equipo multidisciplinario que practica una estricta evaluación clínica, nutricional y la corrección de los parámetros alterados en el perioperatorio. Esta conducta explica el amplio rango observado de días de estadía hospitalaria. Es importante destacar que, aunque se corrigieron los parámetros nutricionales previo al tratamiento quirúrgico, se mantuvo una actitud quirúrgica prudente, al solo extirpar en una primera etapa la mayor extensión de colon dañado (colectomía total), dejando una ileostomía y conservando el recto, con el fin de dar tiempo a la recuperación del estado general del paciente y así evaluar posteriormente el restablecimiento de la continuidad del tubo digestivo, después de al menos 6 meses ( 7 a 76 meses). También influyó en esta conducta el uso frecuente de corticoides en el preoperatorio.

En la CC, la alternativa quirúrgica que se seleccione depende en gran parte de la presencia o ausencia de compromiso del recto o periné. El compromiso de ambas estructuras en conjunto o separadamente contraindican restablecer la continuidad del tubo digestivo usando el recto enfermo en presencia de lesiones perianales o incontinencia. En nuestra serie se practicaron 8 proctocolectomías, todas en pacientes con compromiso rectal, y perineal en 6 de ellos. La presencia de displasia de alto grado o cáncer son también indicaciones de proctocolectomía, que fue la indicación en 1 paciente de esta serie, luego del hallazgo incidental de un carcinoma de recto. A pesar de la extirpación del colon y recto (proctocolectomía) existe el riesgo de una recurrencia en el intestino delgado, lo que se observa con frecuencia variable entre 10 y $30 \% \%^{2,4,9}$. Nosotros lo observamos en solo 1 paciente, con un seguimiento a 75 meses. Probablemente, con el advenimiento de las terapias biológicas esta cifra de recurrencia pueda ser menor, pero estará condicionada por el hábito tabáquico ${ }^{12,13}$.

En esta serie, la colectomía total con ileostomía fue la operación practicada con mayor frecuencia, porque tiene algunas ventajas que merecen ser destacadas: Permite extirpar el colon dañado sin dejar una anastomosis intraabdominal, lo que es particularmente importante en pacientes intervenidos de urgencia por una crisis grave o en aquellos que, aunque intervenidos electivamente, cursan con una forma crónica de la enfermedad y que al momento de la indicación quirúrgica presentan compromiso del estado nutritivo o simultáneamente están inmunocomprometidos por el uso de corticoides, inmunomoduladores o biológicos. Además, esta alternativa quirúrgica, al resecar todo el colon enfermo y preservar el recto, permite en la mayoría de los pacientes suspender el tratamiento inmunosupresor y dar lugar a que el paciente recupere su estado general para evaluar el recto y practicar una IRA ${ }^{5,13}$.

Cuando la enfermedad respeta el recto y el paciente se encuentra en buenas condiciones nutricionales e inmunocompetente se plantea de entrada la IRA. El uso de inmunosupresores o inmunomoduladores al momento de la indicación operatoria podría exponer a los pacientes a una mayor morbilidad infecciosa postoperatoria. Por esta razón, es nuestra práctica suspender este tratamiento, al menos, 3 semanas antes de la operación. Esta situación se presentó en 4 pacientes que recibieron una IRA como primera operación. Además, hubo otros 3 pacientes en quienes se practicó una IRA diferidamente luego de una colectomía total con ileostomía terminal. No registramos reintervenciones por recurrencia en estos pacientes. La experiencia acumulada con IRA en CC muestra cifras de reoperación por recidiva de hasta $30 \%$. Sin embargo, es importante destacar que la IRA permite una adecuada función intestinal y una buena calidad de vida, por lo cual, si bien no se puede considerar como un tratamiento definitivo, estos 2 factores son suficientes para plantearla como una alternativa quirúrgica ${ }^{5,9,13,21}$. Además, la evaluación temprana en el postoperatorio de factores de riesgo de recurrencia, como son el 
tabaquismo, antecedente de fístula, enfermedad perianal o edad de inicio de la enfermedad, permiten considerar y justificar el uso profiláctico de antibióticos, inmunosupresores y eventualmente biológicos, con el fin de evitar o retardar la recurrencia. Datos publicados recientemente permiten concluir que el uso de biológicos e inmunosupresores efectivamente disminuyen la incidencia de recurrencia de la enfermedad, pero sin poder establecer aún los resultados a largo plazo ${ }^{16,22-26}$.

En esta serie, la indicación de fármacos posterior a la cirugía se analizó individualmente, considerando la presencia de los factores de riesgo mencionados. Adicionalmente, es importante señalar que, en los pacientes con IRA, el acceso expedito al recto e íleon terminal facilita el control endoscópico para evaluar la efectividad del tratamiento médico ${ }^{27-29}$. Ante una colitis extensa, sin enfermedad perineal actual ni en la historia previa, que no responde a tratamiento médico, puede considerarse practicar una proctocolectomía con reservorio ileal. Esto implica una detallada información al paciente sobre el riesgo de recurrencia perineal o intestinal que se ha observado en el rango de 25 a $64 \%{ }^{30-32}$.

La colectomía segmentaria es también una opción válida en los casos de compromiso parcelar y estable. En esta serie se practicó en tres pacientes con resultados satisfactorios, concordante con lo comunicado en otros estudios, en los que no observan diferencias con los pacientes sometidos a colectomía total e IRA, pero también advierten que la resección multisegmentaria $(>=2$ segmentos) se acompaña de mayor frecuencia de recurrencia y no es aconsejable $12,15,16$.

La técnica laparoscópica, que se empleó con éxito en la mitad de los pacientes de esta serie, ofrece las ventajas derivadas de un menor trauma a la pared y cavidad abdominal, que se reflejan en una disminución del período de íleo y estadía postoperatoria, como ha sido comunicado previamente $^{3,33}$.

En esta serie, con una mediana de seguimiento de 55 meses, los pacientes usaron menos fármacos luego de la resección quirúrgica, diferencia que, aunque no es significativa $(p=0,44)$ apunta a otro beneficio del tratamiento quirúrgico.

La fortaleza de este estudio radica en comunicar los resultados quirúrgicos en una serie significativa de CC tratada por un equipo multidisciplinario. Sus principales limitaciones son el carácter retrospectivo, el reclutamiento de pacientes que recibieron tratamiento médico en otras instituciones, la falta de registro del hábito tabáquico y los potenciales sesgos derivados de la revisión de registro clínicos.

En conclusión, en esta serie, la morbilidad es similar a la comunicada por centros de experiencia en el tratamiento quirúrgico de las enfermedades inflamatorias intestinales. La mayoría de los pacientes necesitó de una colectomía total para el control de la enfermedad y una ileostomía, que fue definitiva en la mitad de ellos.

\section{Referencias}

1. Lapidus A, Bernell O, Hellers G, Lofberg R. Clinical course of colorectal Crohn's disease: A 35-year follow-up study of 507 patients. Gastroenterology 1998; 114 (6): 1151-60.

2. Bernell O, Lapidus A, Hellers G. Recurrence After Colectomy in Crohn's Colitis. Dis Colon Rectum 2001; 44: 647-54.

3. da Luz Moreira A, Stocchi L, Remzi FH, Geisler D, Hammel J, Fazio VW. Laparoscopic surgery for patients with Crohn's colitis: a case-matched study. J Gastrointest Surg 2007; 11 (11): 1529-33.

4. Handler M, Dotan I, Klausner JM, Yanai H, Neeman E, Tulchinsky H. Clinical recurrence and re-resection rates after extensive vs. segmental colectomy in Crohn's colitis: a retrospective cohort study. Tech Coloproctol 2016; 20 (5): 287-92.

5. Li Y, Stocchi L, Mu X, Cherla D, Remzi FH. Long-term Outcomes of Sphincter-Saving Procedures for Diffuse Crohn's Disease of the Large Bowel. Dis Colon Rectum 2016; 59 (12): 1183-90.

6. Polle SW, Slors JFM, Weverling GJ, Gouma DJ, Hommes DW, Bemelman WA. Recurrence after segmental resection for colonic Crohn's disease. Br J Surg 2005; 92 (9): 1143-9.

7. Tekkis PP, Purkayastha S, Lanitis S, Athanasiou T, Heriot AG, Orchard TR, et al. A comparison of segmental vs subtotal/total colectomy for colonic Crohn's disease: A meta-analysis. Colon Dis 2006; 8 (2): 82-90.

8. Moghadamyeghaneh Z, Carmichael JC, Mills SD, Pigazzi A, Stamos MJ. Outcomes of Bowel Resection in Patients with Crohn's Disease. Am Surg 2015; 81 (10): 1021-7.

9. Fichera A, McCormack R, Rubin MA, Hurst RD, Michelassi F. Long-term outcome of surgically treated Crohn's colitis: A prospective study. Dis Colon Rectum 2005; 48 (5): 963-9. 
10. Mills S, Stamos MJ. Colonic Crohn's Disease. Clinics 2007; 1 (212): 309-13.

11. Strong S, Steele SR, Boutrous M, Bordineau L, Chun J, Stewart DB, et al. Clinical Practice Guideline for the Surgical Management of Crohn's Disease. Dis Colon Rectum 2015; 58 (11): 1021-36.

12. Martel P, Betton PO, Gallot D, Malafosse M. Crohn's colitis: Experience with segmental resections; results in a series of 84 patients. J Am Coll Surg 2002; 194 (4): 448-53.

13. Riordan JMO, Connor BIO, Huang H, Victor JC, Gryfe M, Macrae HM, et al. Long-term Outcome of Colectomy and Ileorectal Anastomosis for Crohn's Colitis 2011; 1347-54.

14. Martin S, Vogel J. Restorative procedures in colonic crohn disease. Clin Colon Rectal Surg 2013; 26 (2): 100-5.

15. Kiran RP, Nisar PJ, Church JM, Fazio VW. The Role of Primary Surgical Procedure in Maintaining Intestinal Continuity for Patients With Crohn s Colitis. Ann Surg 2011; 253 (6): 1130-5.

16. Lee JL, Yu CS, Lim S-B, Park IJ, Yoon YS, Kim CW, et al. Surgical Treatment of Crohn Colitis Involving More Than 2 Colonic Segments. Medicine (Baltimore) 2016; 95 (22): e3793.

17. Zúñiga Á, Zárate A, Fullerton D, Duarte I, Álvarez M, Quintana C. Evolución de los pacientes con enfermedad de Crohn de colon operados con el diagnóstico de colitis ulcerosa. Rev Chil Cirugía 2008; 60 (1): 35-40.

18. Dindo D, Demartines N, Clavien P-A. Classification of Surgical Complications. Ann Surg 2004; 240 (2): 205-13.

19. Silverberg MS, Satsangi J, Ahmad T, Arnott IDR, Bernstein CN, Brant SR, et al. Toward an integrated clinical, molecular and serological classification of inflammatory bowel disease: report of a Working Party of the 2005 Montreal World Congress of Gastroenterology. Can J Gastroenterol 2005 Sep [cited 2017 May 9]; 19 Suppl A: 5A-36A.

20. Yamamoto T, Watanabe T. Surgery for luminal Crohn's disease. World J Gastroenterol 2014; 20 (1): 78-90.

21. Elton C, Makin G, Hitos K, Cohen CRG. Mortality, morbidity and functional outcome after ileorectal anastomosis. Br J Surg 2003; 90 (1): 59-65.

22. Nguyen GC, Nugent Z, Shaw S, Bernstein CN. Outcomes of patients with Crohn's disease improved from
1988 to 2008 and were associated with increased specialist care. Gastroenterology 2011; 141 (1): 90-7.

23. Rungoe C, Langholz E, Andersson M, Basit S, Nielsen NM, Wohlfahrt J, et al. Changes in medical treatment and surgery rates in inflammatory bowel disease: a nationwide cohort study 1979-2011. Gut 2014; 63 (10): 1607-16.

24. Vaughn BP, Moss AC. Prevention of post-operative recurrence of Crohn's disease. World J Gastroenterol 2014; 20 (5): 1147-54.

25. Carla-Moreau A, Paul S, Roblin X, Genin C, Peyrin-Biroulet $\mathrm{L}$. Prevention and treatment of postoperative Crohn's disease recurrence with anti-TNF therapy: A meta-analysis of controlled trials. Dig Liver Dis 2015; 47 (3): 191-6.

26. O'Connor A, Hamlin PJ, Taylor J, Selinger C, Scott N, Ford AC. Postoperative prophylaxis in Crohn's disease after intestinal resection: a retrospective analysis. Frontline Gastroenterol 2017; 8 (3): 203-9.

27. Harb WJ. Crohn's Disease of the Colon, Rectum, and Anus. Surg Clin North Am 2015; 95 (6): 1195-210.

28. Baumgart DC, Sandborn WJ. Crohn's disease. Lancet 2012; 380 (9853): 1590-605.

29. Buisson A, Chevaux J-B, Allen PB, Bommelaer G, Peyrin-Biroulet L. Review article: the natural history of postoperative Crohn's disease recurrence. Aliment Pharmacol Ther 2012 Mar [cited 2017 May 9]; 35 (6): 625-33.

30. Melton GB, Fazio VW, Kiran RP, He J, Lavery IC, Shen $B$, et al. Long-Term Outcomes With Ileal Pouch-Anal Anastomosis and Crohn s Disease. Trans. Meet Am Surg Assoc 2008; 126 (4): 251-9.

31. Kariv Y, Remzi FH, Strong SA, Hammel JP, Preen M, Fazio VW. Ileal Pouch Rectal Anastomosis: A Viable Alternative to Permanent Ileostomy in Crohn's Proctocolitis Patients. J Am Coll Surg 2009; 208 (3): 390-9.

32. Turina M, Remzi FH. The J-pouch for Patients with Crohn's Disease and Indeterminate Colitis: (When) Is it an Option? J Gastrointest Surg. 2014; 18 (7): 13434 .

33. Umanskiy K, Malhotra G, Chase A, Rubin M, Hurst RD, Fichera A. Laparoscopic colectomy for Crohn's colitis. A large prospective comparative study. J Gastrointest Surg 2010; 14 (4): 658-63. 